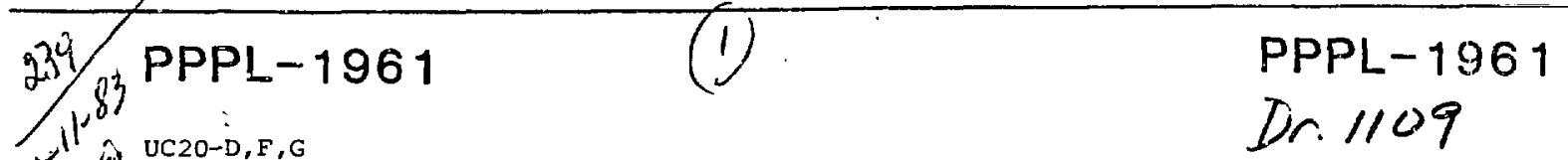

(AC2O-D,F,G

PIPL--1961

DE83 005051

RADIO-FREQUENCY WAVE TRAJECTORIES FOR

CURRENT DRIVE IN TOKAMAK REACTORS

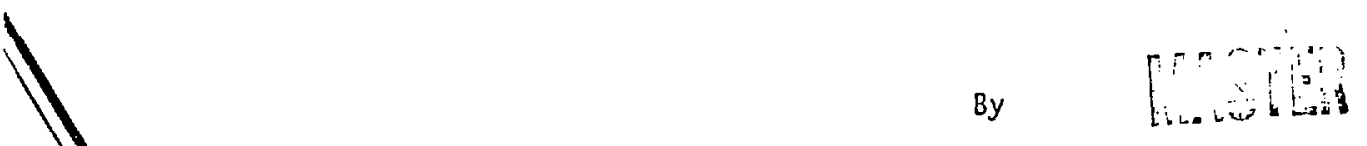

K.L. Wong and M. Ono

DECEMBER 1982

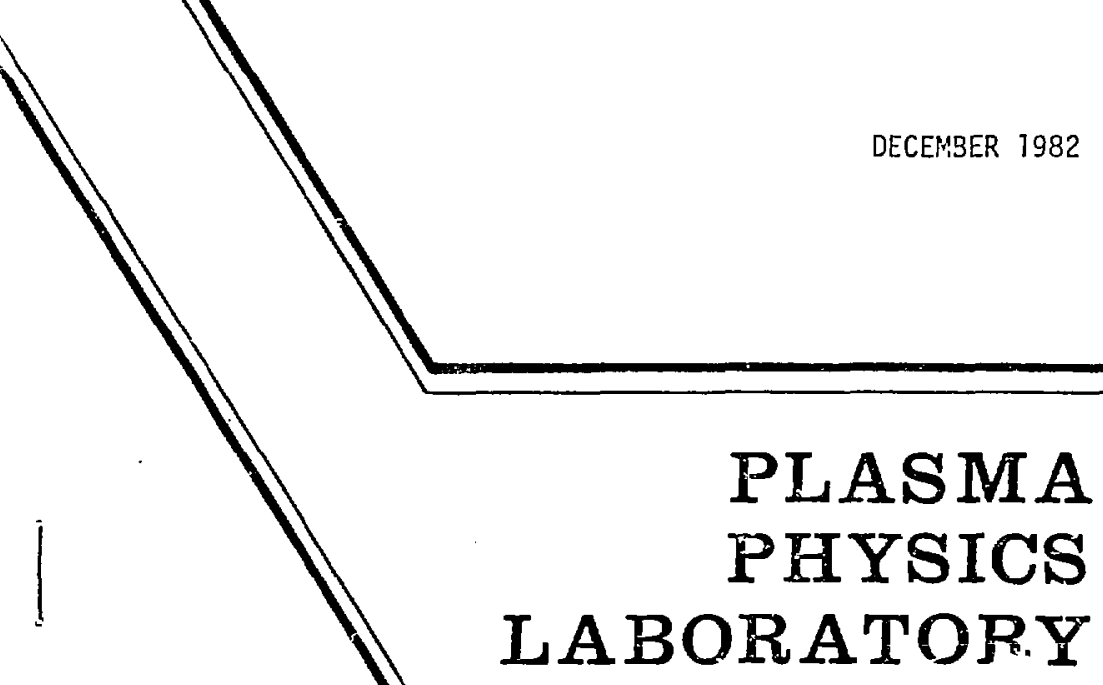




\title{
Radio-Frequency Wave Trajectories for \\ Current Drive in Tokamak Reactors
}

\section{King-Iap Wong and Masayuki ono}

Princeton Plasma Physics Laboratory, Princeton University Princeton, New Jersey 08544

\begin{abstract}
Detailed ray tracing calculations were carried out for three modes of waveguide-launched radio-frequency wave: for tokamak reactor parameters to evaluate their applicabi. ty for steady state current drive. The merits and demerits of each mode are discussed.
\end{abstract}




\section{Introduction}

The success of lower hybrid wave current drivel has rapidly grown out of small experimental devices ${ }^{2-4}$ into large tokamaks, $5-10$ causing plasma current generation with radio-frequency waves to become one of the major topics in fusion research. This is primarily due to the strong interest in steady state tokamaks. Until recently, most of the current drive experiments have utilized lower hybrid waves. This trend has been prompted by readily available equipment originally planned for lower hybrid wave heating where the waveguide phase can be easily adjusted to launch an unidirectional wave. So far, they have been successful only in low density plasmas. It is well known that magnetized plasmas can support many modes of waves and their suitability for sustaining a steady state tokamak reactor can be expected to differ from mode to mode. The major advantages of lower hybrid waves consist of the all-metal waveguide coupler (phased waveguide arrays) and the relatively low wave frequency $\leq 10$ GHz) so that multi-megawatt radio-fregsency technology is already available without major development. These advantages are shared by at least two other modes: the fast wave ${ }^{11}$ (whistler) and the ion Bernstein wave, ${ }^{2}$ both of which can also be used to drive currents via electron Landau damping. Since the density limit of lower hybrid wave current drive is not well understood, 10 it is not clear that they can be used in tokamak reactors. Therefore, it is important to consider other modes of plasma waves. In this paper, we present de'siled ray tracing 
calculations for the lower hybrid wave, fast wave, and ion Bernstein wave with reactor-like parameters and compare the merits of these modes in terms of wave accessibility and efficiency.

\section{Ray Tracing Code}

The ray tracing computer $\operatorname{code}^{13}$ follows the wave trajectories by numerically integrating the following equations: 14

$$
\begin{aligned}
& \frac{d \vec{r}}{d t}=-\frac{\partial D}{\partial \vec{k}} / \frac{\partial D}{\partial \omega}, \\
& \frac{d \vec{k}}{d t}=+\frac{\partial D}{\partial \vec{r}} / \frac{\partial D}{\partial \omega},
\end{aligned}
$$

The conventional toroidal coordinates $(r, \theta, \phi)$ are used with the assumption of concentric circular magnetic surfaces. $\omega$ and $\vec{k}$ de:?te the wave angular frequency and wave number which satisfy the local electromagnetic wave dispersion relation: 15

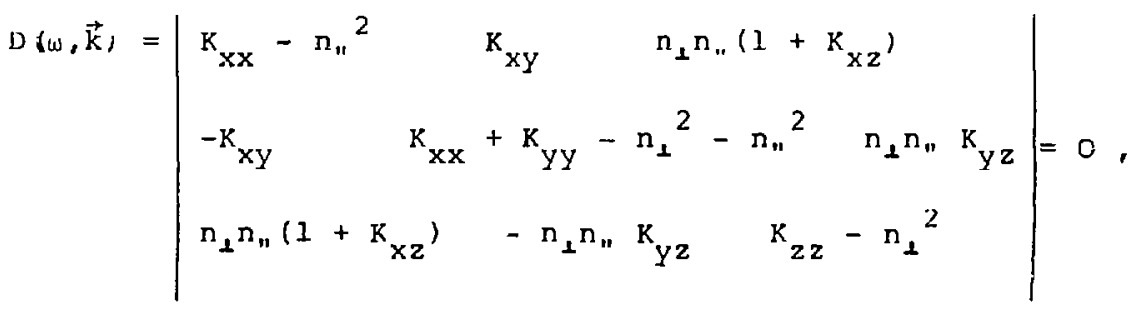

where $n \equiv k c / \omega$ and ", I denote components paraliel and perpendicular to the magnetic field $(\vec{B}=\hat{B z})$, respectively. The $k_{i j}$ are various elements of the hot plasma dielectric tensor ${ }^{15}$ with the finite-Larmor-radius terms included. For the parameters of our 
interest, we can put $\mathrm{K}_{\mathrm{xz}}=0$. The chosen plasma parameters are similar to those of the proposed FED device as shown in the following:

$$
\begin{aligned}
& \text { major radius: } \quad R_{0}=458 \mathrm{~cm} \\
& \text { minor radius: } \quad \mathrm{a}=120 \mathrm{~cm} \\
& \begin{array}{l}
\text { toroidal magnetic } \quad B_{0}=50 \mathrm{kG} \\
\text { field: }
\end{array} \\
& \text { peak electron density: } n_{0}=2 \times 10^{14} \mathrm{~cm}^{-3} \\
& \begin{array}{l}
\text { electron density } \quad n(r)=n_{0}\left[0.01+0.99 \cos \left(\frac{\pi}{2} \cdot \frac{r}{a}\right)\right] \\
\text { protile: }
\end{array} \\
& \text { deuterium density: } \quad n_{1}(r)=n(r) / 2 \\
& \text { tritium density: } \quad n_{2}(r)=n(r) / 2 \\
& \text { safety factor: } \quad \underline{q}(0)=1.0 \quad(r=0) \\
& q(a)=1.8 \text { or } 3.8 \quad(x=a) \\
& \text { peak electron 'I'eo }=15 \mathrm{keV} \\
& \text { temperature: } \\
& \text { electron temperature } \\
& \text { profile: } \\
& T_{e}(r)=T_{e c}\left[0.99\left(1-\frac{r^{2}}{a^{2}}\right)^{2 / 30(q(a)-1)}+0.01\right] \\
& \text { ion temperature: } \quad T_{i}(r)=T_{e}(r) \\
& B_{y}(r)=\frac{B_{0} a^{2} /(r q(a))}{R_{0}+r \cos \theta} \cdot\left[1-\left(1-\frac{r^{2} q(a)}{a^{2}}\right)^{q}\right]
\end{aligned}
$$

In order to sustain a steady state tokamak with these parameters via electron Landau damping of plasma waves, good wave 
penetration into the plasma core with $n_{11} \sim 1.2-1.5$ is necessary.

III. Lower Hybrid Waves

In a cold plasma the lower nybrid wave dispersion relation is:

$$
1-\frac{\omega_{p i}^{2}}{\omega^{2}}-\frac{k_{1}^{2}}{k^{2}} \cdot \frac{\omega_{p e}^{2}}{\omega^{2}}+\frac{k_{L}^{2}}{k^{2}} \frac{\omega^{2}}{\omega_{c e}^{2}}=0 .
$$

It is an electrostatic wave such that

$$
\frac{E_{n}}{E_{\perp}}=\frac{k_{n}}{k_{\perp}}
$$

In slab geometry the wave can reach the lower hybrid resonance if it satisfies the accessibility condition: 16

$$
\mathrm{n}^{2}{ }^{2}>1+\frac{w_{p e}^{2}}{w_{c e}^{2}},
$$

which obviously tavors low density and high magnetic field. The situation can be quite different in the presence of a rotational transtorm. In a tokamak magnetic field, $n_{\text {. }}$ varies along the wave trajectory ${ }^{1 /}$ and waves launched at the plasma edge may not reach the lower hybric resonance even when they satisfy kq. (6). This can be qualitatively illustratea as follows :

$$
k_{n}=k_{\theta} \cdot \frac{{ }_{\theta}^{B}}{B}+k_{\phi} \frac{{ }_{\phi}^{B}}{B},
$$

where the subscripts $\theta$ and $\phi$ denote the poloidal and toroidal directions, respectively. 
Put $B_{\phi}=B_{0} R_{0} /\left(R_{0}+r \cos \theta\right)$ and assume $1 / q^{2} \cdot r^{2} /\left(R_{0}+r \cos \theta\right)^{2}<1$, Irom Eq. (2), Eq. (4) and Eq. (7), one can easily show that

$$
\frac{d k_{n}}{d t}=\frac{\omega}{q} \frac{\omega_{p e^{2}}}{\omega_{c e}^{2}} \frac{r \sin \theta}{\left(R_{o}+r \cos \theta\right)^{2}} /\left(1+\omega_{\left.p e^{2} / \omega_{c e}{ }^{2}\right)}\right.
$$

Therefore, $n_{1}=k_{\text {, }} c / \omega$ varies along the wave trajectory. Ray tracing results in Fig. la show that $n_{\text {" }}$ oscillates as one would expect from Eq. (8). The power deposition profile is shown in Fig. In for two values of $n_{t}=k_{\phi} c / \omega(1.2$ and 1.5$)$. There is only a minor ditference between the two cases because wave absorption occurs mainly near the peak value of $n_{n}$ along the wave trajectory (which is higher than the value of $n_{n}$ launched at the plasma surface). Over $y 0 \%$ of the wave power is absorbed within the region $50 \mathrm{~cm}<r<90 \mathrm{~cm}$ via electron Landau damping. Figure $l c$ and $F i g$. Ld show the ray trajectory projected on the poloidal and the toroidal planes. It is apparent that as the wave propagates into the plasma, the perpendicular wavelength gets shorter as the density increases, and the radial penetration is very slow. One may think intuitively that lower hybrid waves should be able to penetrate deeper into the plasma at higher frequencies because of the higher perpendicular group velocity. Ray tracing results show that this is not necessarily true in a tokamak magnetic field. Figure 2 shows the region of wave absorption for varlous wave trequencies. 'l'he optimum frequency for the chosen plasma parameters is about $1 \mathrm{GHz}$, and wave penetration becomes worse at 
higher frequencies. This obviously contradicts our intuition. Wave penetration at higher frequencies is somewhat improved by raising $q(a)$. Careful examination of the wave parameters along its trajectory reveals that the radial group velocity $v_{\text {gr }}$ is actually smaller for a higher wave frequency. In order to understand this behavior, let us put $R=R_{0}+r \cos \theta$. $k_{\theta}=m / r, k_{\phi}=n / R_{i}$ from the cold plasma wave dispersion relation, we can obtain 17

$$
\begin{aligned}
& \frac{d k_{\theta}}{d t}=\frac{I}{r} \frac{\partial D}{\partial \theta}, \frac{\partial D}{\partial \omega}=-\frac{\omega \sin \theta}{R}
\end{aligned}
$$

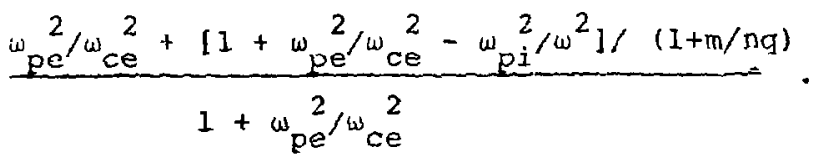

It is apparent Erom Eq. (9) that $k_{\theta}$ can get large at high frequencies. Since

$$
k_{1}=\left(k^{2}-k_{n}{ }^{2}\right)^{1 / 2}=\left(k_{\theta}{ }^{2}+k_{r}{ }^{2}\right)^{1 / 2} \simeq k_{n} \omega_{p e} / \omega=n_{n} \omega_{p e} / c .
$$

$k_{\perp}$ is almost independent of $w$ at a fixed value of $n_{\text {, }}$ and $a$ larger $k_{\theta}$ would yield a smaller $k_{r}$ which can result in a lower group velocity in the radial direction. Ray tracing results indicate that raising the lower hybrid wave frequency from $1.15 \mathrm{GHz}$ to $2 \mathrm{GHz}$ reduces the radial group velocity by a factor 
of ten. The wave at $2 \mathrm{GHz}$ mainly propagates along the poloidal direction and damped near the plasma surface due to the slow radial penetration.

At very high wave frequencies ( $\geq 5 \mathrm{GHz}$ ), we find that instead of surface absorption, the wave is reflected near the plasma edge without damping. This happens because $k_{\theta}$ gets so large that it forces $k_{r}$ to zero as shown in Fig. 3a. This would cause a reflection in the radial direction. When the reflected wave reaches the plasma edge, part of it will tunnel out of the plasma and interact with the vacuum chamber wall while the rest of it will be reflected back towards the plasma interior. One can follow the rays after many reflections, but one cannot control $n_{n}$, especially for the waves reflected from

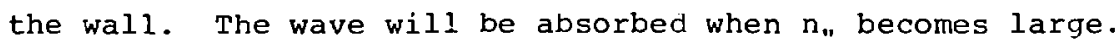

All the results presented up to now were obtained with initial $k_{\theta}$ set equal to zero. One can launch the wave with an appropriate $k_{\theta}$ so that $k_{r}$ never goes to zero and the reflection can be avoided as shown in Fig. $3 b$ and Fig. 3c. Another way to avoid reflection is to raise the magnetic field (Fig. 4). Yet neither of these methods can make the wave penetrate to the plasma center. From these results we can see that it is marginal to rely on lower hybrid waves to sustain a steady state tokamak reactor unless one can change the plasma parameters for better wave penetration, namely, lower the temperature and density and raise the magnetic field. It should be pointed cut here that the wave power deposition profiles presented thus far were 
obtained by assuming linear electron Landau damping due to a locally Maxwellian distribution function. In order to drive currents, quasi-linear effects should take place which flatten the slope of the distribution function, lower the damping rate, and improve the wave penetration. ${ }^{18}$

\section{Fast Waves}

In a cold magnetized plasma, the fast wave dispersion relatior can be expressed in a simple form: 19

$$
\omega \simeq \frac{k^{2} c^{2}}{\omega_{p e}^{2}} \omega_{c e}|\cos \alpha|
$$

provided that $\omega_{\mathrm{Ci}} / \cos \alpha \ll \omega \ll \omega_{\mathrm{LH}}^{2} \cos \alpha / \omega_{\mathrm{Ci}}$ where $\alpha$ is the angle between $\vec{k}$ and $\vec{B}, i . e ., k_{n}=k \cos \alpha, k_{\perp}=k \sin \alpha$. In a slab geometry where $k_{1}=k_{x}, \vec{B}=B \hat{z}$, the group velocity of the wave is:

$$
\vec{v}_{g}=\frac{\partial u}{\partial \vec{k}}=\frac{w}{k}\left[\sin \alpha, 0, \frac{1+\cos ^{2} \alpha}{\cos \alpha}\right],
$$

and the wave polarization is:

$$
\vec{E}=E_{\mathbf{X}}\left[1, i|\cos \alpha|, \frac{\omega}{\omega c e} \sin \alpha \frac{|\cos \alpha|}{\cos \alpha}\right] \text {. }
$$

The wave energy density is:

$$
w_{0}=\frac{1}{8 \pi}\left|E_{x}\right|^{2} n_{. .}^{2}
$$

Fast waves with $n_{\text {" }} \sim(1)$ are mainly damped by electron Landau damping. Wave damping via transit time magnetic pumping is 
negligible. In order to compare the effectiveness of current drive with fast waves vs lower hybrid waves, let us start with the one-dimensional quasi-linear diffusion equation:

$$
\frac{\partial f}{\partial t}+\frac{e}{m} E \frac{\partial f}{\partial v}=\frac{\partial}{\partial v}\left(D \frac{\partial f}{\partial v}\right)+\left.\frac{\partial f}{\partial t}\right|_{C}
$$

where $E=$ static electric tield parallel to $\vec{B}$

$D \simeq \pi(e / m)^{2} k_{n} / \omega \frac{E_{n}^{2}}{\Delta k_{.}}$

$E_{n}=$ component of rf electric field parallel to $\vec{B}$

$\Delta k_{n}=$ wiath of $\mathrm{rt}$ power spectrum

$\left.\frac{\partial f}{\partial t}\right|_{C}=\begin{aligned} & \text { coliision term whose torm depends on the collision } \\ & \text { model. }\end{aligned}$

The steady state solution without static electric field is:

$$
\frac{\partial f}{\partial v}=-\left.\frac{1}{D} \int_{v} \frac{\partial f}{\partial t}\right|_{c} d v
$$

Therefore, the slope of the electron distribution function is proportional to $I, D \propto 1 / E_{.}{ }^{2}$ and one has a pertect plateau if $D \rightarrow \infty$. The effectiveness of a current ariver via electron Landau damping can be characterized by the factor $E \equiv D /$ (wave power flux). A wave with a small value of $E$ will have weak electron Landau damping so that a large power flux is needed for a given value of $\mathrm{D}$. If Landau damping is the only dissipation mechanism and the wave power spectrim remains unchanged inside the plasma, then the ratio of current densily to power dissipation $\left(J / P_{\alpha}\right)$ is independent of $E$; but usually there are other competing dissipation mechanisms and a small $\varepsilon$ may become undesirable. The value of $E$ Jepends on the wave 
polarization and the group velocity which can be calculated from the dispersion relation. From Eq. (4) and Eq. (11) we can show that for reactor parameters,

$$
\frac{\varepsilon \text { (Fast Wave) }}{E \text { (Lower Hybrid Wave) }}=\frac{\omega_{p e}^{2}}{\omega_{c e}^{2}} \frac{1}{n_{n}^{2}} \frac{\sin ^{2} \alpha}{1+\cos ^{2} \alpha} \sim 0.1-1 .
$$

This means that fast waves can be almost as effective as lower hybrid waves for current drive in tokamak reactors.

Detailed ray tracing has been carried out in a toroidal geometry with the same set of plasma parameters as is listed In Section II. vue to the rotational transform, n, varies along the wave trajectory as shown in Fig. 5a. Since the fast wave, by definition, has a longer perpendicular wave length compared with the slow wave, the wave penetrates faster into the plasma interior as shown $1 \mathrm{n}$ Fig. $5 \mathrm{~b}$ and Fig. 5c, but the power deposition profile in Fig. 5d is quite similar to the lower hybrid wave case (see Fig. 2d). Figure 6 shows the wave absorption region at various frequencies. 'the optimum frequency is about $1 \mathrm{GHz}$ and penetration at higher frequencies improves with higher $q$ values. The similarity between the fast wave and the slow wave is not so surprising it one realizes that they can convert to each other and the wave characteristics should be similar near the mode conversion region. Figure $7 a$ shows the tamiliar $k_{\perp}$ vs $r$ curves for the illustration of mode conversion in a slab model. In a tokamak magnetic fieid, the situation is complicated by the rotational transform and the toroidal effect. Figure $7 b$ shows $k_{\perp}$ vs 5 cbtained from ray tracing calculations. One can see that lower hybric waves 
and fast waves almost coalesce at $r-60 \mathrm{~cm}$ where 998 of the wave energy has been dissipated.

Despite the similarities in the optimum wave absorption profile, the fast wave behaves quite different]y from the lower nybrid wave in some other respects. When the fast wave is launched with initial $\mathrm{k}_{\theta}=0$ at the plasma edge, $\mathrm{k}_{\theta}$ remains small $\left(k_{\theta} \ll k_{r}\right)$, unlike lower hybrid waves, untii the wave approaches the center of the plasma. When the wave is near the plasma center, $k_{\theta}$ can be large and this results in a reflection in the radial direction. Another important difference between the fast wave and the lower hybrid wave is the exceilent penetration of fast waves at low frequencies (f $<1 \mathrm{GHz}$, see Fig. 6). At $f-000 \mathrm{MHz}$ the wave can reach the plasma center, undergo a reflection, and propagate outward wich very little damping. Stronger damping would occur at a higher plasna temperature. Therefore, if one wants to operate a steaky state tokamak at a peak temperatıre of $\sim 25 \mathrm{keV}$, fast wave currerst drive at this freguency range may be a viable option.

\section{$\mathrm{V}$ Ion Bernstein Waves}

The ion Bernstein wave ${ }^{12}$ is a hot Dlasma wave which exists because of the finite ion Larmor radius effect. At low densities where $\omega_{p e}^{2} / \omega^{2} \ll n_{\perp}^{2}$, the wave is electrostatic and the dispersion reiation in a slab geometry is:

$$
\mathrm{n}_{1}^{2} \mathrm{~K}_{\mathrm{xx}}+\mathrm{n}_{11}^{2} \mathrm{~K}_{\mathrm{zz}}=0
$$

At high densities the wave becomes guasi-electrostatic, in that the energy density stored in the wave magnetic field become: comparable to or even larger than in the wave 
electric field, and one must use Eq. (3) for an accurate description of the wave dispersion relation. Uniike lower hybrid waves, ion Bernstein waves with a wide range of $n_{n}$, have been shown previously to penetrate very easily into a hot-dense plasma. 13 one can calculate the efficiency factor for ion Bernstein wave ass 근 nain

$$
\frac{E(I B W)}{E(L W W)}=10^{-5}\left(k_{\perp} \rho_{i}\right)^{7}
$$

The $k_{\perp} \rho_{i}$ term was included to account for the resonance effect where $k_{\mathfrak{1}} \rho_{\dot{i}}^{+\infty}$. Ion Bernstein wave is launched from the low field edge of the plasma where $k_{\perp} \rho_{i} \leq 1$ and propagated toward the ion cyclotron harmonic resonance. Therefore, the efficiency fartor (or damping) which is negligible can increase rapidly near the resonance and reach $\mathscr{G}(1)$ for $k_{1} \rho_{i} \geq 6$. This effect causes the wave which is initially undamped to damp strongly via electron Landau damping near the resonance layer which an be placed near the center of the plasma. In a reactor like high temperature situation $\left(\mathrm{T}_{e}>10 \mathrm{keV}\right)$, the damping is usually dominated by electron Landau damping iather than the ion cyclotron harmonic damping. As can be seen from Fig. 8a, the strong wave damping near the plasma center is due to this enhanced electron Landau damping near the second harmonic cyclotron resonance of the tritium ions. If there is no cyclotron harmonic layer inside the plasma, the wave can shine through with very little absorption. A somewhat surprising result 
from ray tracing in a tokamak magnetic field is that $n_{\text {" changes }}$ sign along the wave trajectory as shown in Fig. 8b. This would generate current in both directions and greatly reduce its effectiveness. Figure 9 shows the real and the imaginary

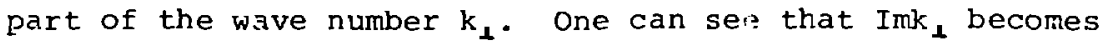
large when ithe ion Bernstein wave approaches the second cyclotron harmonic layer of the tritium ions (located at $r=0$ ). The gap between the spikes is the region ..n which $n_{\text {, goes through }}$ zero and changes sign because Landau damping cannot occur when $\left|n_{11}\right|<1$. Ion Bernstein waves, therefore, can be used to sustain a steady state tokamak only if we start out with an asymmetric electron distribution function so that only waves propagating in the preferred direction are äamped and drive current. There is no need to launch unidirectional waves in such a current drive scheme.

VI. Summary and Discussion

We have analyzed the wave trajectories of the lowar hybrid wave, fast wave, and ion Bernstein wave for steady state reactor parameters. The results are quite different from slab model calculations because of the rotational transform and the toroidal effects. It is found that each of the three modes has its own problems even under ideal situations. The lower hybrid wave is the most effective mode to plateau the electron distribution function, but it has difficulty reaching the 
fiasma center. Fast waves have good penetration only at low frequencies, which results in weak damping unless the electron temperature is very high. Ion Bernstein waves can penetrate very well, particularly for low $n_{n}$. The difficulty lies in the fact that, for the ion Bernstein wave, $n_{n}$ changes sign along its trajectory.

In this paper, we calculate the linear propagation and damping of waves in a 508 deuterium and tritium plasma. Nonlinear effects, plasma density fluctuations, $\alpha$-particle, and other impurity effects are not included. Since we confine ourselves to waveguide-launched waves, other low frequency modes like Alfven waves 20 are not considered. It is interesting to note that Alfven waves can be driven unstable by a-particles. 21 One may be able to launch an unidirectional Alfven wave, from an external source, and let the plasma amplify the wave and sustain the current. In this way most of the current drive power would come directly from the $\alpha$-particles. This scheme substantially reduces the circulacing power in the reactor, making it really attractive. This possibility will be a topic for future studies.

\section{Acknowledgments}

The authors would like to thank Dr. W. Hooke, Dr. D. Ignat, Dr. F. Perkins, Dr. T. H. Stix and Dr. E. Valeo for helpful discussions. This work was supported by the U.S. Bepartment of Energy Contract No. DE-ACO2-76-CHO3073. 


\section{REFERENCES}

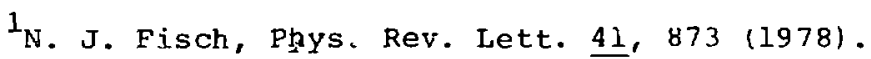

${ }^{2}$ K. L. Wong, Phys. Rev. Lett. 43,438 (1979).

${ }^{3}$ R. MCWilliams, E. J. Valeo, R. W. Motley, W. M. Hooke, and L. Olsen, Phys. Rev. Lett. $\underline{44}, 245$ (1980).

${ }^{4} \mathrm{~K}$. L. Wong, R. Horton and M. Ono, Phys. Rev. Lett. 45,117 $(1980)$.

5. Yamamoto, 'I'. Imai, M. Shimada, N. Suzuki, M. Maeno, S. Konoshima, T. Fujii, K. Uehara, T. Nagashima, A. Funahasni and N. Fujisawa, Phys. Rev, Lett. 45, 716 (i980).

6. Nakamura, T. Cho, S. Kubo, T. Shimozuma, H. Kawai, K. Yamazaki, T. Maekawa, Y. Terumichi, Y. Hamada, and S. Tanaka, Pnys: Rev. Lett. 47, 1902 (1981).

${ }^{7} \mathrm{~S}$. C. Luckhardt, M. Porkolab, S. F. Knowlton, K.-T. Chen, A. S. Fisher, F. S. McDermott, and M. Mayberry, Phys. Rev. Lett. $48,152(1982)$.

$8_{\text {K. Ohkubo, S. Takamura, K. Kawahata, T. Tetsuka, K. Matsuura, }}$ N. Noda, K. Sakurai, S. Tanahashi, and J. Fujita, Nucl. Fusion $22,203(1982)$.

${ }^{9}$ M. Porkolab, J. J. Schuss et al., Proceedings of the International Conference on Plasma Physics and Nuclear Fusion Research (Baltimore, September 1982), paper IAEA-CN-41/C-4. 
${ }^{3.0}$ S. Bernabei, C. Daughney, P. Efthimion, W. Hooke, J. Hosea, F. Jobes, A. Martin, E. Mazzucato, E. Meservey, R. Motley, J. Stevens, S. Von Goeller, and R. Wilson, Phys. Rer. Lett 49, $1255(1982)$.

${ }^{11} \mathrm{D}$. W. Ignat, Proceedings of the 4 th Topical Conference on Radio-Frequency Plasma Heating (February 1981). The University of Texas, Austin, Texas, paper C-12.

$12 \mathrm{M}$. Ono and K. L. Wong, Phys. Rev. Lett. 45,1105 (1980).

$13 \mathrm{M}$. Ono, ‥ H. Stix. K. L. Wong, and R. Horton, in proceedings of the 2nd Joint Grenoble-Varenna International Symposium of Heating in Toroidal plasmas (Commission of the European Communities. Directorate-General XII-Fusion Programme. Brussels, 1980) Vol. I, p 593.

14 T. H. Stix, Theory of Plasmá Waves (McGraw-Hill, New York, 1962) p. 58 .

${ }^{15}$ J. E. Sharer, J. B. Beyer, D. T. Blackfield, and T. K. Mau, Nucl. Fusion 19, 1171. (1979).

${ }^{16}$ V. E. Golant, Soviet Physics-Tech. Phys. 16, 1980 (1972).

17 D. W. Ignat, Phys. Fluids 24, 1110 (1981).

${ }^{18}$ F. J. Paoloni, R. W. Motley, W. M. Hooke, and S. Bernabei, Phys. Rev. Lett. 39, 1081 (1277).

19A. Bers, C. F. F. Karney, and K. Th-ilhaber, RLE Frogress Report (Massachusetts Institute of Technology) No. 115, p. 186 (January 1975). 
${ }^{2 C}$ R. A. Demirkhanov, A. G. Kirov, L. F. Ruchko, and A. V. Sukachev, JETP Lett. 33, 28 (1981).

${ }^{21}$ M. N. Rosenbluth and $P=H=$ Rutherford, Phas. 몬. Let: 34 , 1428 (1975); also K. T. T'sang, D. J. Sigmar and J. C. Whitson, Phys. FIuids 24, 1508 (1981). 


\section{FIGURE CAPTIONS}

Fig. 1. Characteristics of lower hybrid waves in a tokamak. (a) Variation of the parallel index of reflection along the wave trajectory. (b) Wave absorption profile at two initial values of $n_{t}$. (c) wave trajectory projected on a poloidal plane. (d) Wave trajectory projected on a toroidal plane.

Fig. 2. Locations of the wave absorption region for lower hybrid waves at yarious Erequencies. $P$ denotes the fraction of power remained in the wave when reflection takes place. (a) $q(a)=1.8$, (b) $q(a)=3.8$.

Fig. 3. Reflection of high frequency (5 GH:) lower hybrid vaves near the plasma edge, (a) $k_{r}$ is forced to zero when $k_{\theta}$ increases, the wave is launched with $k_{\theta_{0}}=0$. (b) When the wave is launched with $k_{\theta 0}=0.42 \mathrm{~cm}^{-1}, \mathrm{k}_{r}$ never goes to zero and reflection does not occur. (c) Wave absorption profile for $k_{\theta 0}=0.42 \mathrm{~cm}^{-1}$.

Fig. 4. $5 \mathrm{GHz}$ lower hybrid waves at a higher magnetic field $\left(\mathrm{B}_{\mathrm{O}}=75 \mathrm{kG}\right)$. (a) $\mathrm{k}_{\mathrm{r}}$ never goes to zero so that reflection does not occur. (b) Wave absorption profile.

Fig. 5. Characteristics of fast waves in a tokamak (a) variation of the parallel index of reflection along the wave trajectory. (b) Wave absorption profile. (c) Wave 
trajectory projected on a poloidal plane. (d) Wave trajectory projected on a toroidal plane.

Fig. 6. Location of wave absorption region for fast waves at various frequencies. $P$ denotes the fraction of power remaining in the wave when reflection takes place. (a) $q(a)=1.8$, (b) $q(a)=3.8$.

Fig. 7. (a) Schematic of mode conversion between lower hybrid waves and fast waves in a slab geometry. (b) Variation of $k_{\perp}$ from ray tracing calculations of $1.15 \mathrm{GHz}$ lower hybrid waves and fast waves.

Fig. 8. Characteristics of ion Bernstein waves (f: $=50.6 \mathrm{MHz}$, $\left.\mathrm{n}_{t}=1.4, \mathrm{q}(\mathrm{a})=1.8\right)$. (a) wave absorption profile. note that $\mathrm{f}=2 \mathrm{f}_{\mathrm{CT}}$ at $\mathrm{r}=0$. (b) variation of $\mathrm{n}_{n}$ along the wave trajectory. (c) Wave trajectory projected on a poioidal plane. (d) wave trajectory projected on a toroidal plane.

Fig. 9. Real and imaginary parts of the perpendicular wave number $k_{\perp}$ for ion Bernstein waves with the same parameters as those in Fig. 8 . 

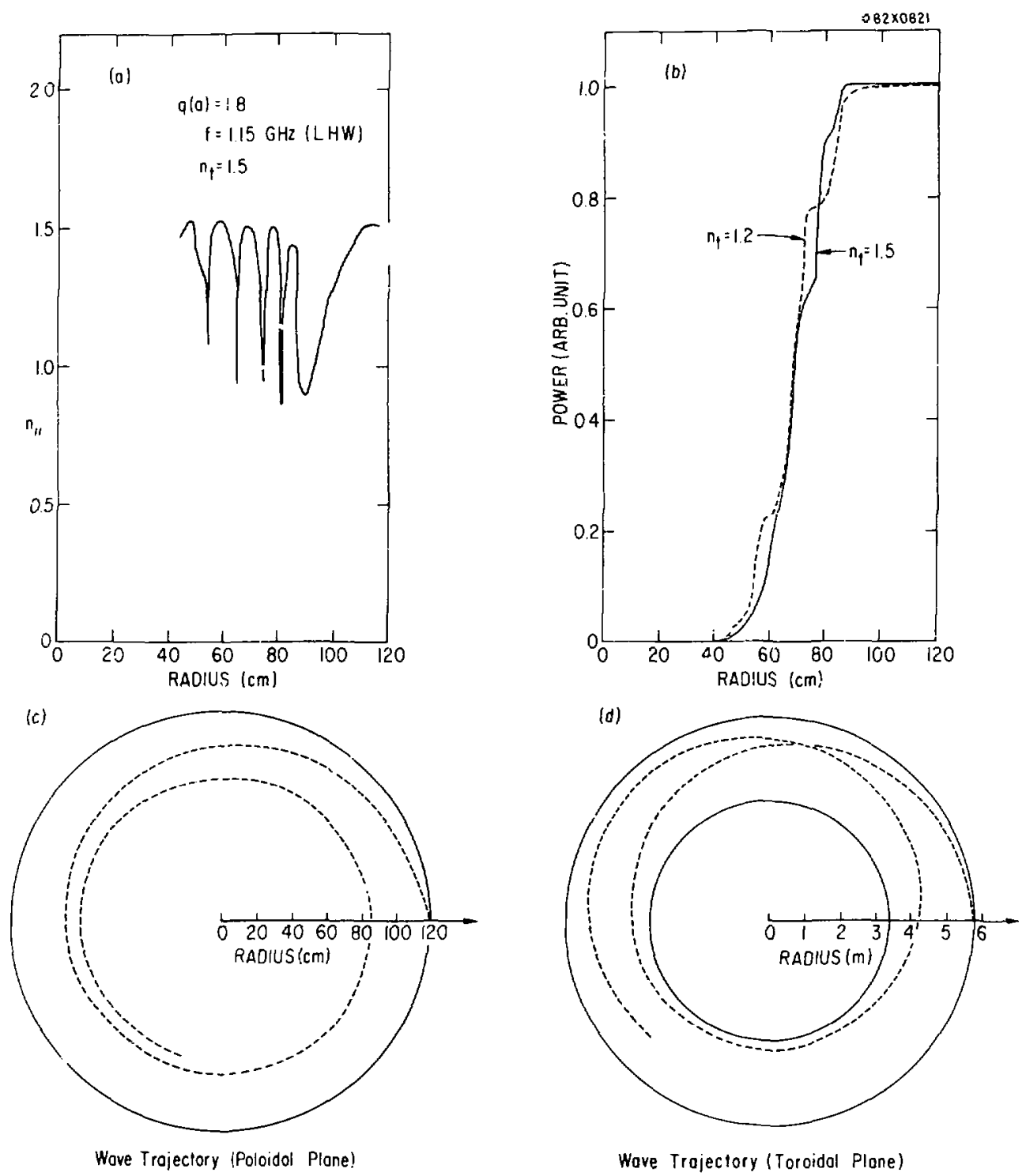

Fig. I 

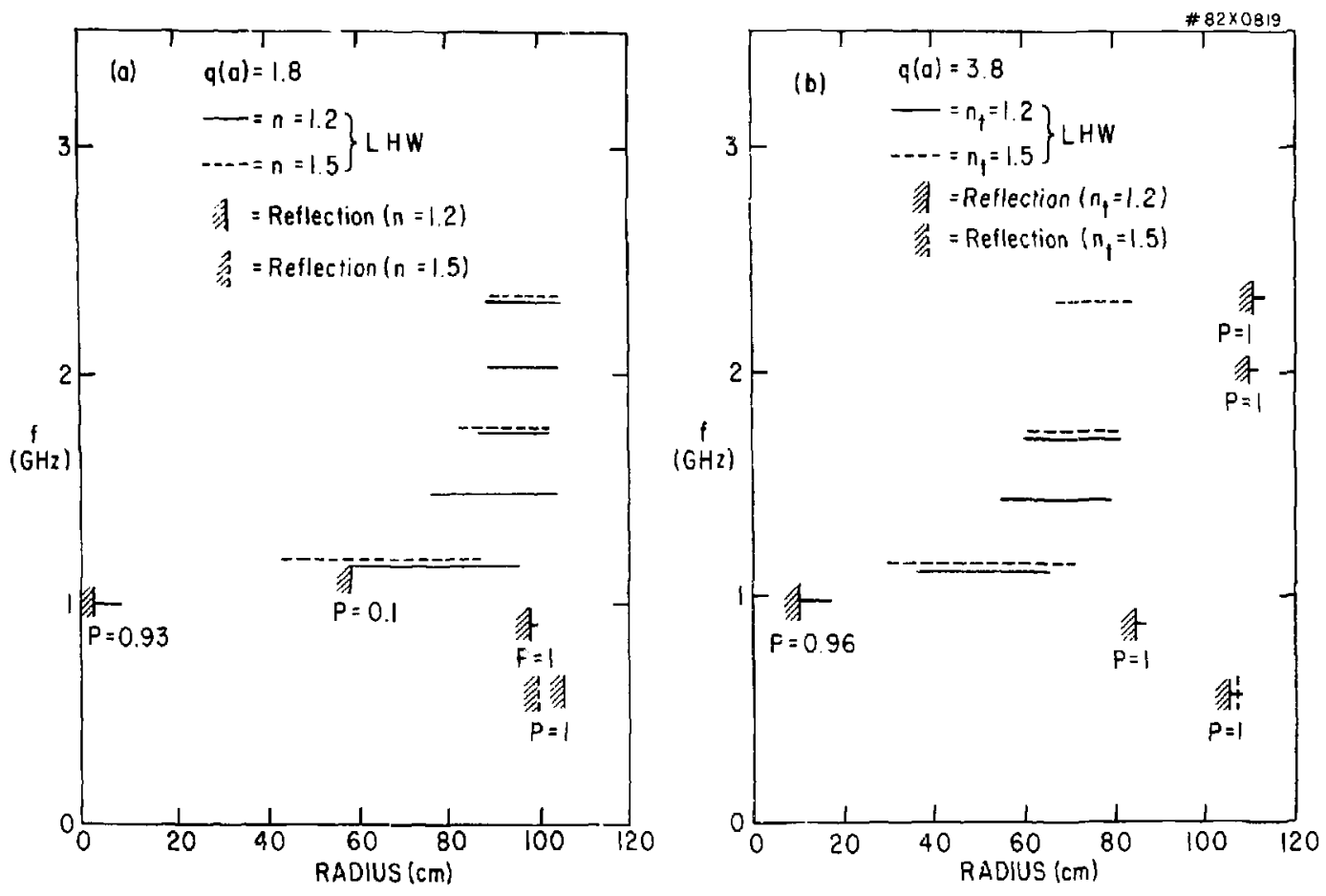

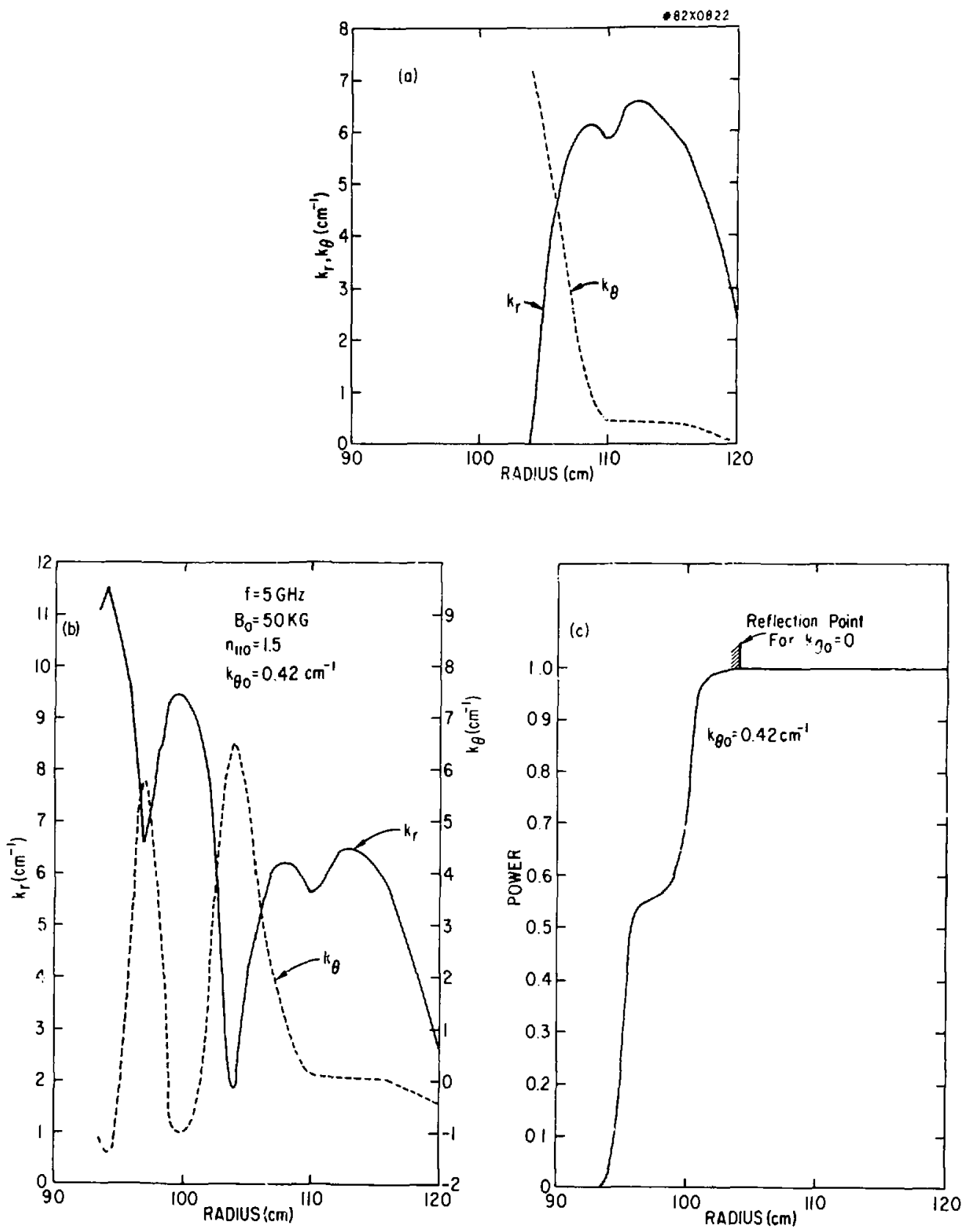

Fig. 3 

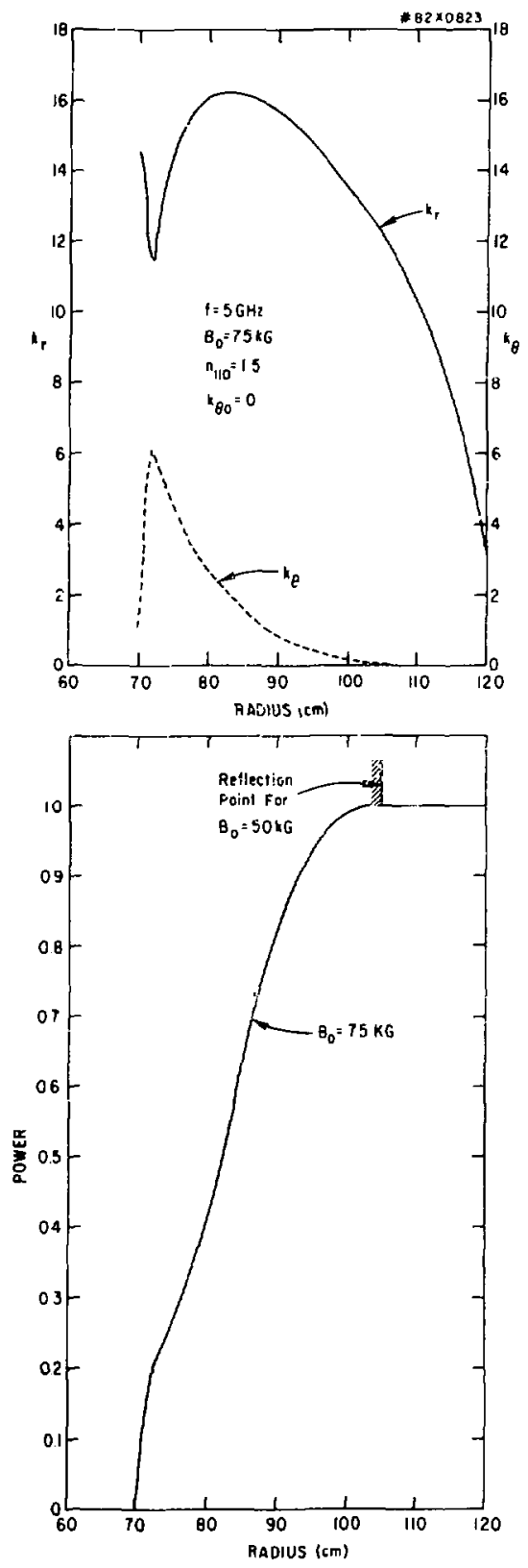

Fig. 4 

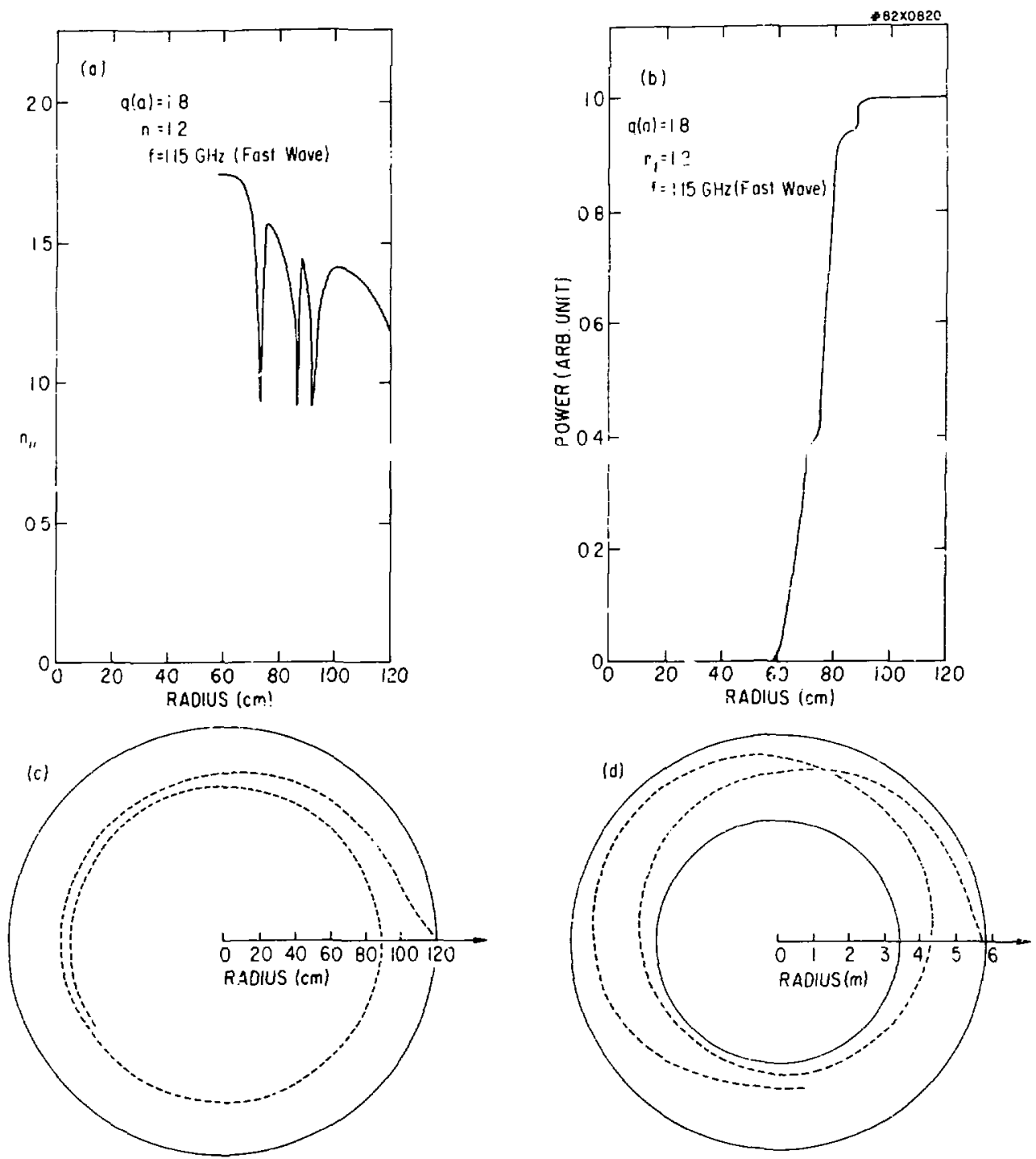

Wave Trojectory (Poloidal Plane)

Wave Trojectory (Toroidal Plane)

Fig. 5

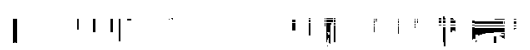



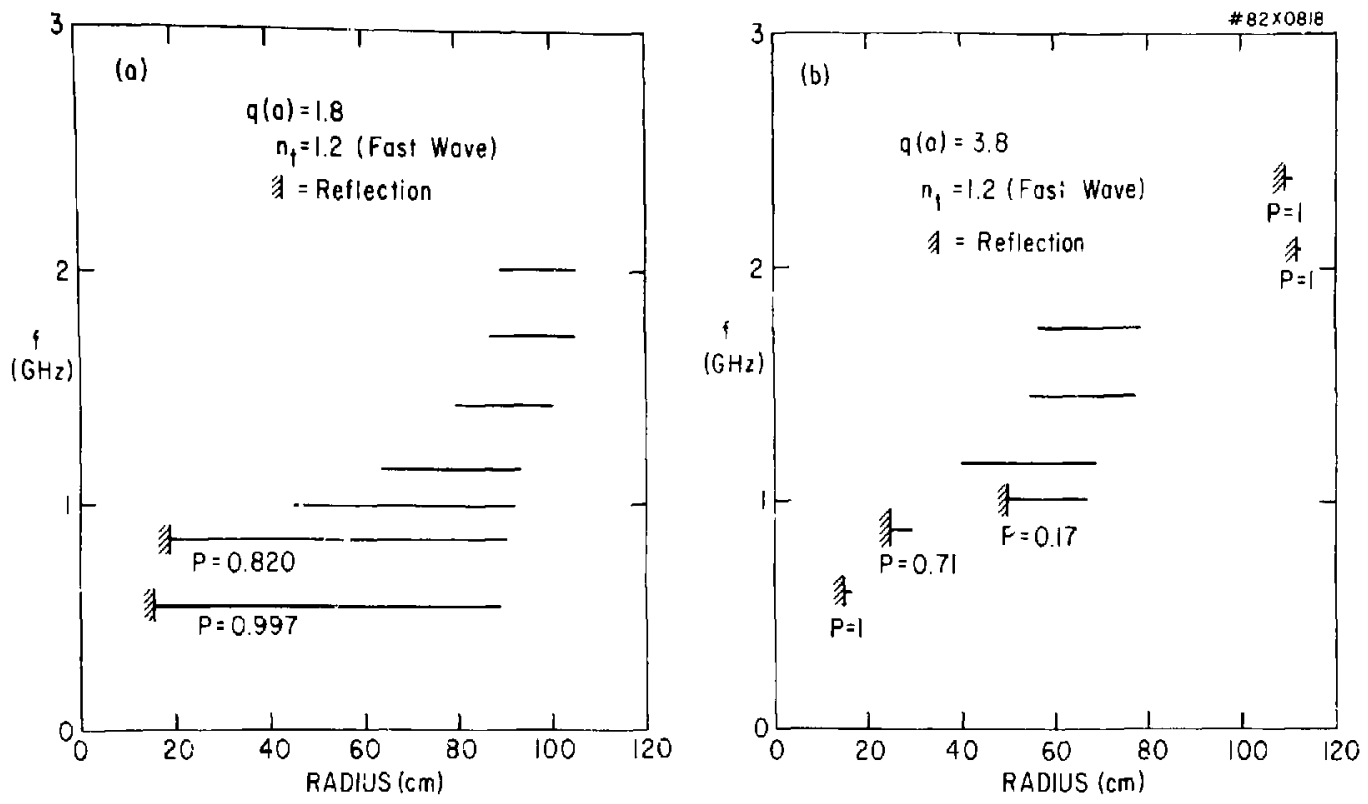

ชั

Fig. 6 

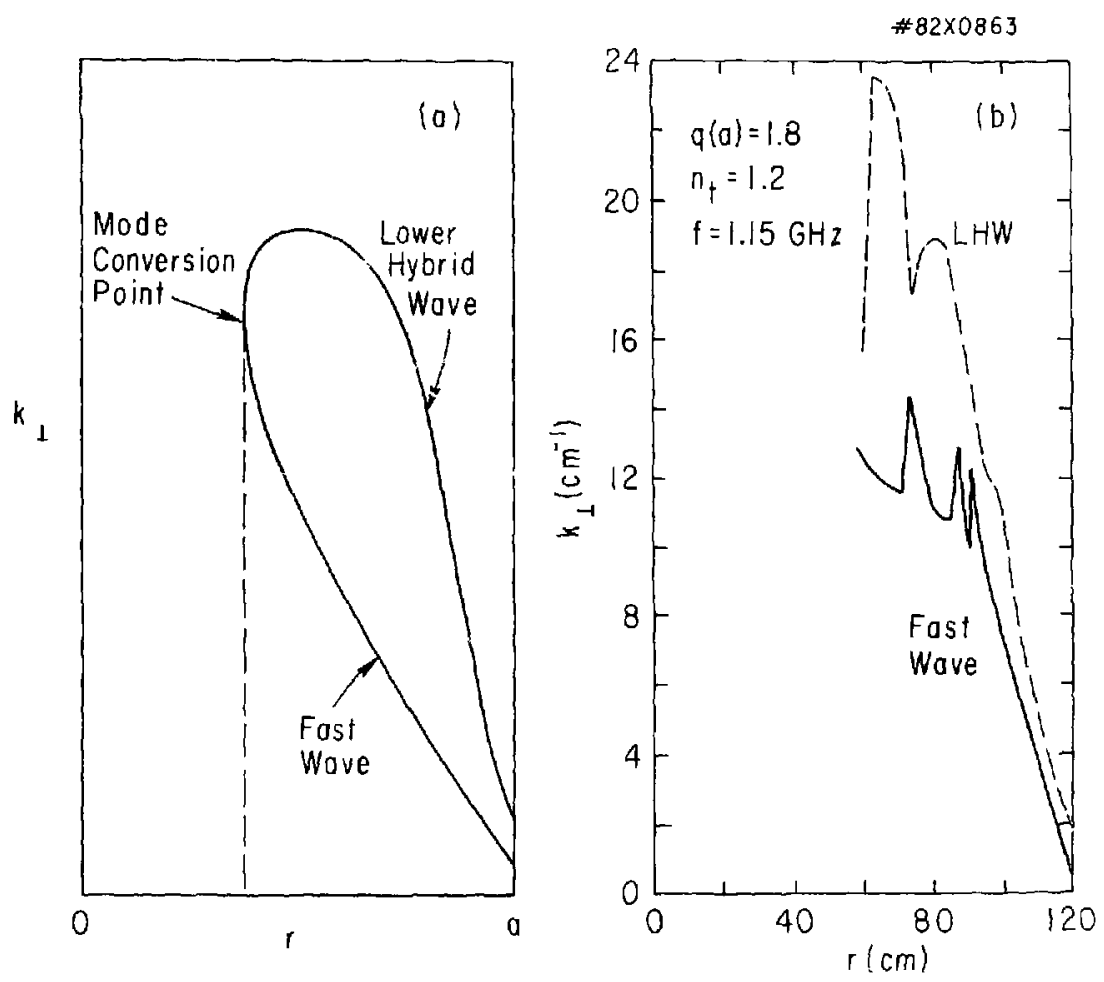

$N$

Fig. 7 


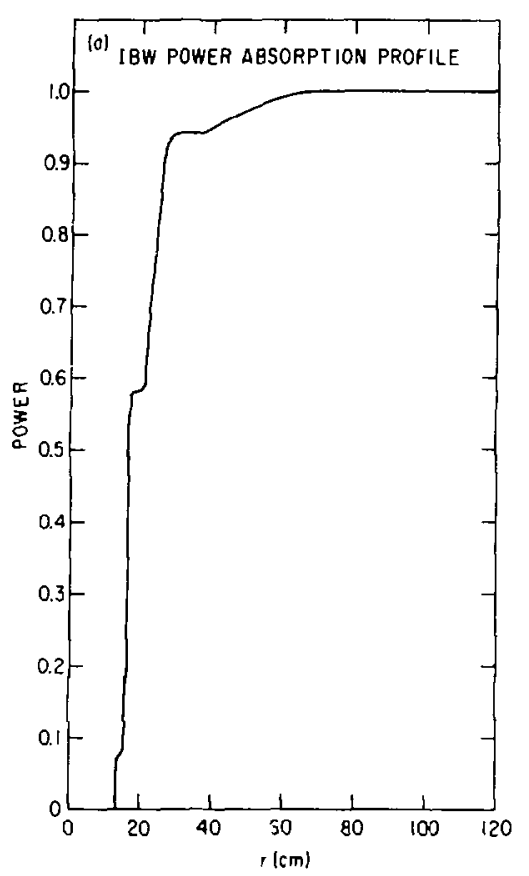

(c)

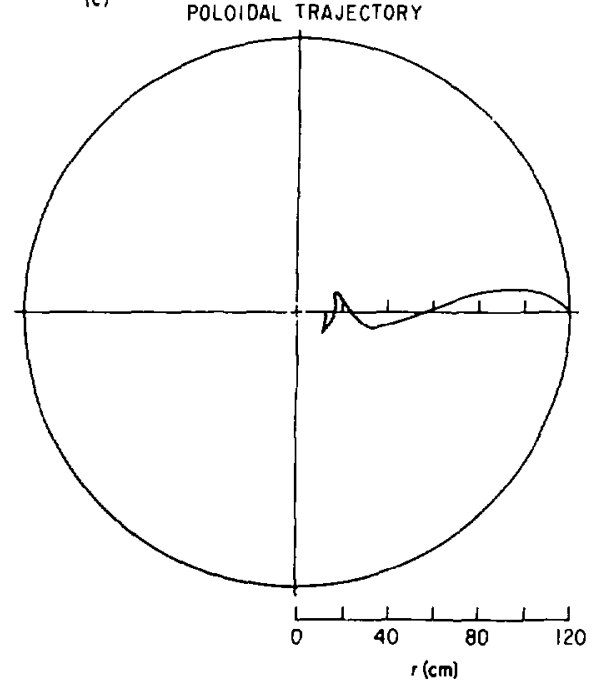

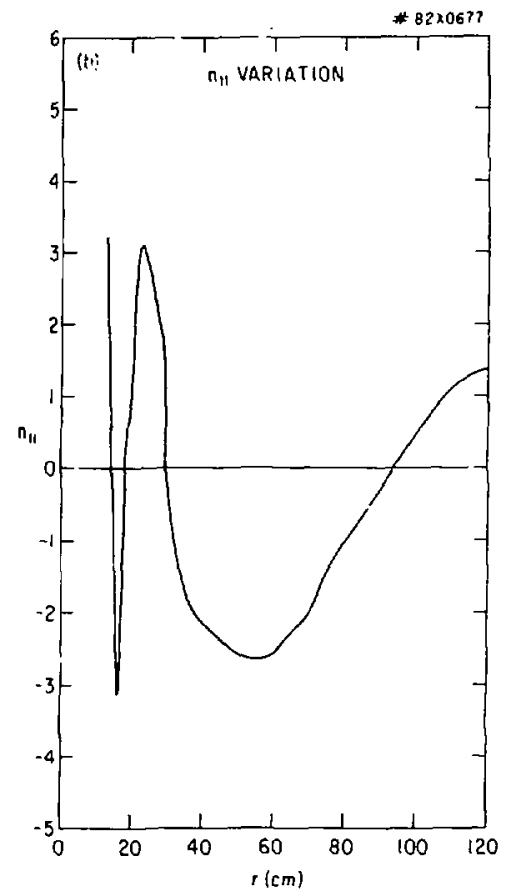

(d) TOROIOAL TRAJECTORY

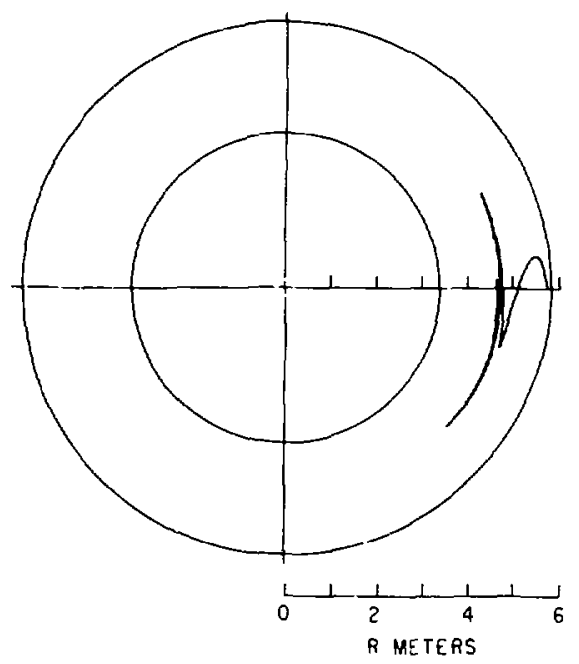

Fis. 8 


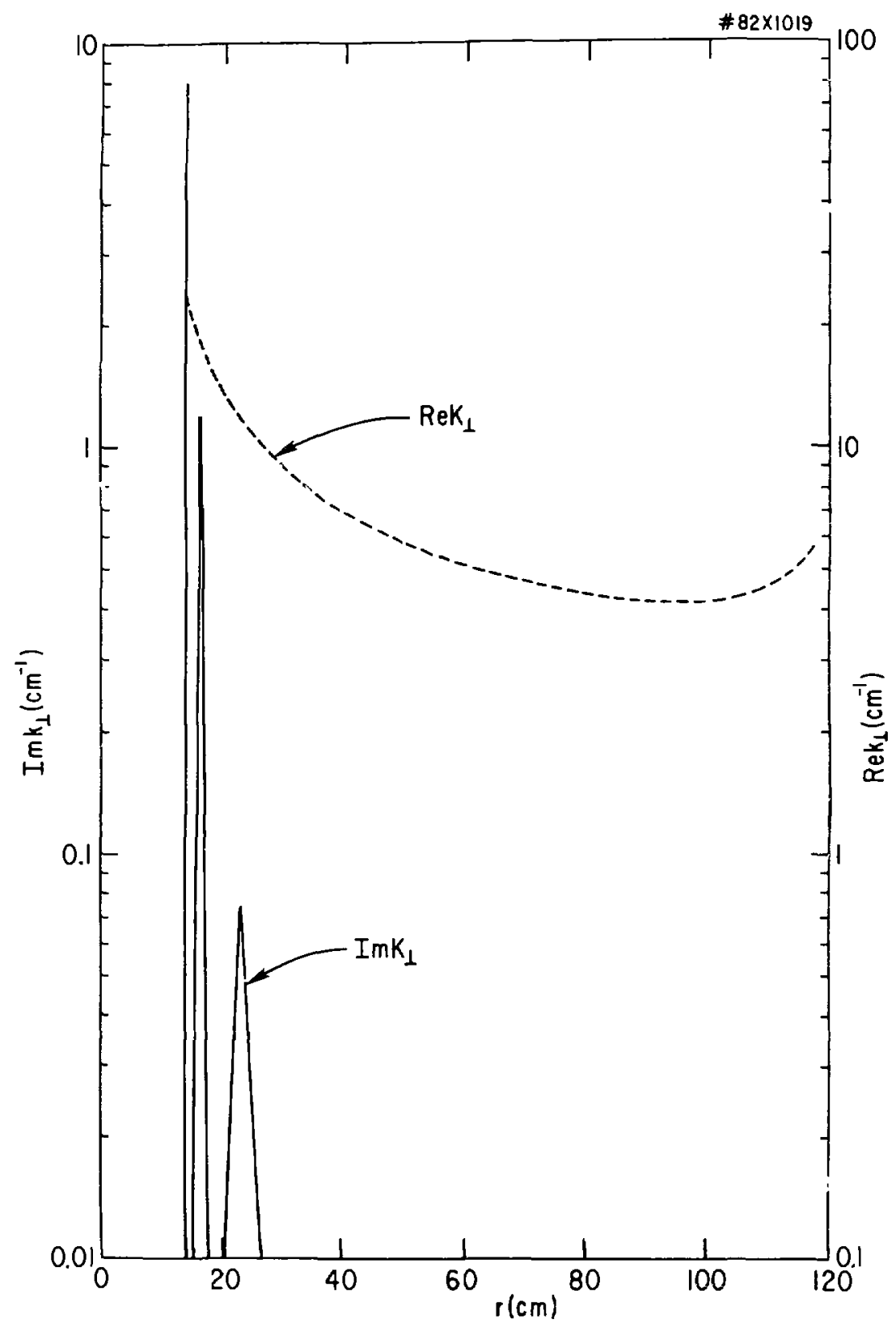

Fig. 9 